\title{
Use of Ursodeoxycholic Acid on Post-menopausal Obesity, Hepatic steatosis and Plasma Profile as an Alternative Treatment for Hormone Replacement Therapy
}

\author{
Angelo Christiano Franzoni, Aryane Martins Amorim, João Victor Mariano da Silva, João \\ Augusto Pires Storti and Monique Cristine de Oliveira* \\ Departamento de Medicina-Bioquímica; Faculdade Ingá; Maringá-PR-Brasil.
}

\begin{abstract}
The aim of this study was to evaluate the efficacy of the ursodeoxycholic acid (UDCA) as an alternative treatment for menopause pathologies. For this, female Swiss-CD-1 mice were ovariectomized (OVX); SHAM operated mice served as controls. Ten weeks after operation, each group of mice was treated during four weeks with either UDCA, or 17ß-estradiol+progesterone. The blood and tissues (liver and periuterine adipose tissue - PUAT) were harvested. The OVX mice had increase body weight, accompanied by liver lipid accumulation. UDCA and HRT treatments reduced these parameters. PUAT was also increased in OVX group and UDCA and HRT treatments reduced this. Histology confirmed the results in the liver and PUAT. The plasma glucose levels were elevated in the OVX mice, as well as total cholesterol, LDL cholesterol and VLDL cholesterol levels and reduced in OVX-UDCA and OVX-HRT. These results suggest that UDCA could reduce weight gain and fat liver deposition caused by ovariectomy, such as HRT, showing a possible alternative treatment for post-menopausal metabolic dysfunctions with UDCA.
\end{abstract}

Key words: liver disease, menopause, obesity, ursodeoxycholic acid

\section{INTRODUCTION}

The climacteric, or menopause is characterized as a transition period between the reproductive phase, or menarche and non-reproductive phase. These alterations are associated with the endocrine hormonal changes related to ovarian function, causing dysfunction, loss of fertility and weakened menstrual cycle. Due to these hormonal changes, a woman enters in menopause period usually around 51 years old and varies during this period by hormone deficiency, as well as physiological and behavioural changes.

Decreased circulating estrogen levels have several physiological consequences, including important changes in the metabolism that result in altered body fat distribution and increased risk of metabolic syndrome (Reis et al. 2003; Cooke and Naaz 2004; Schneider et al. 2006), with subsequent development of non-alcoholic fatty liver disease (NAFLD) in women (Suzuki and Abdelmalek 2009). NAFLD is emerging as a risk factor for other diseases related to accumulation of fat in tissues and blood, especially cardiovascular diseases (Villanova et al. 2005; Lobo 2008). In addition, estrogen deficiency can lead to variations in the distribution of body fat, leading to obesity due to the lipolytic action of this hormone, with greater accumulation of abdominal fat.

\footnotetext{
*Author for correspondence: prof.moniqueoliveira@uninga.edu.br
} 
The combined estrogen and progesterone therapy is commonly used in the form of hormone replacement therapy (HRT) (Volpe et al. 1986), which prevents weight gain and skin folds, observed after menopause (Hassager and Christiansen 1989). Concerns over the safety of HRT have, however, grown with several findings. The Heart and Estrogen/progestin Replacement Study showed a short-term increase in cardiovascular risk in the women selected for being at high risk of cardiovascular disease (Hulley et al. 1998), and the Million Women Study reported a significant association of the use of HRT with breast cancer (Beral 2003). Consistent with these findings, the Women's Health Initiative (WHI) (Rossouw et al. 2002; Anderson et al. 2004) found an increased risk of coronary heart disease and breast cancer for the women given HRT. However, according to the International Menopause Society, properly timed HRT is safe for healthy women in their early postmenopause (Lekander et al. 2009).

Thus, although the efficacy of HRT is well documented in the literature. Several side effects resulting from the use of HRT stimulated the search for improved therapies involving the variety of means related to obesity and metabolic disorders. Therapies studying the pathways involved in the obesity and metabolic disorders have shown that the ursodeoxycholic acid (UDCA) is used for the treatment of diseases related to liver disorders, especially cholestasis, obesity and lipemic frames (Hofmann 1994). UDCA is a hydrophilic bile acid constituent of most of Chinese black bear bile (Colombo et al. 1996; Keiding et al. 1997). In humans, however, it is present in small amounts as a secondary bile acid (Colombo et al. 1996), representing 3\% of the total pool of bile (Keiding et al. 1997). UDCA has cytoprotective, immunomodulatory and antiapoptotic properties, therefore reduces the rate of cholesterol molecules absorption in the intestine, thus regulating the metabolism (Pageaux et al. 1995), and diseases such as NAFLD may also be significantly improved by the treatment with UDCA.

Thus, the aim of this study was to evaluate the effects of UDCA as an alternative treatment for menopause pathologies comparing to the effects of hormone replacement therapy (estrogen + progesterone) helping to elucidate the mechanisms involved post-menopausal disorders.

\section{MATERIAL AND METHODS}

\section{Animals}

Female Swiss CD1 mice weighing 25-30 g (seven weeks of age) were randomly assigned to shamoperation (control - SHAM), or bilateral ovarietomy (OVX). For surgery, mice were anesthetized with a mixture of ketamine-xylazine $(50-10 \mathrm{mg} / \mathrm{kg}$ i.p.), and submitted to trichotomy and antisepsis of the abdomen. Then, a transverse incision was made (approximately $1 \mathrm{~cm}$ ). The ovaries were removed after ligation of the tubes. The sham-operated mice had their ovaries exposed but not removed. Body weight (BW) and food intake were monitored biweekly. Animals were fed ad libitum with a standard laboratory diet $\left(\mathrm{Nuvilab}^{\circledR}\right.$, São Paulo, Brazil) for 10 weeks. Then, these animals (17 weeks of age) were subdivided in six groups $(\mathrm{n}=8)$. Group 1-SHAM (control, vehicle arabic gum), 2-SHAM-HRT (control treated with estrogen and progesterone - hormone replacement therapy), 3-SHAM-UDCA (control treated with ursodeoxycholic acid), 4-OVX (ovariectomized, vechicle arabic gum), 5-OVXHRT (ovariectomized treated with estrogen and progesterone - hormone replacement therapy), 6OVX-UDCA (ovariectomized treated with UDCA). The animals were treated via gavage, according to the body weight of each mouse for 30 days. The HRT (17- $\beta$-estradiol + progesterone4-pregnene-3,20-dione) groups received $0.01+0.1$ $\mathrm{mg} / \mathrm{kg} / \mathrm{d}$ and UDCA groups received $15 \mathrm{mg} / \mathrm{kg} / \mathrm{d}$. Periuterine adipose tissue (PUAT) and liver were removed and weighed. The substrates for treatment were purchased from Sigma Chemical Co. (St. Louis, USA). All the experiments were conducted in strict adherence to the guidelines of the Ethics Committee for Animal Experimentation of the State University of Maringá (079/2008).

\section{Analysis of Serum Lipid Profile and Glycaemia} Mice (21 weeks of age) were fasted for $12 \mathrm{~h}$ and blood was collect by cardiac puncture. Plasma glucose concentrations were determined using a glucose analyzer $\left(\right.$ Optium $\left.^{\circledR}\right)$. Total cholesterol and triglycerides (TG) were analysed by standard methods (Gold Analisa ${ }^{\circledR}$ ). VLDL-cholesterol was calculated from TG levels (Friedewald's equation) and LDL-cholesterol was determined by subtracting HDL and VLDL from the total cholesterol. 


\section{Histological Analyses and Total Lipid Content Measurements}

Small pieces of dissected periuterine adipose tissue (PUAT) and from left lobe of the liver were fixed in $4 \%$ formaldehyde, dehydrated in series of increasing concentrations of alcohol, cleared in xylen and embedded in paraffin to perform histological sections $(7 \mu \mathrm{m})$, and stained with hematoxylin and eosin (HE), which showed cellular morphology. The total lipid contents of the liver were measured by the gravimetric method, through the homogenization of liver fragments in a 2:1 chloroform-methanol mixture (Folch et al. 1957).

\section{Treatment of Data}

Data are expressed as means \pm standard error (SEM). Significant differences among the means were identified by one-way ANOVA, followed by a Tukey post-test. The results are given in the text as probability values $(P)$ with $p<0.05$, adopted as a criterion of significance. Statistical analysis was conducted through the GraphPAD software.

\section{RESULTS}

\section{Body Weight, Tissue Weights and Food Intake}

As confirmation of successful OVX-induced suppression of endogenous oestrogen production, at 14 weeks after operation, the OVX groups exhibited an evident uterine atrophy, with uterine/BW ratios three times lower than SHAMoperated animals (Table 1).
In the OVX mice, the body weight was higher in $70 \%$ in relation to SHAM group and this was accompanied by increased adiposity, as could be seen by the differences in PUAT/BW ratio $(+202 \%)$. UDCA-treatment and HRT-treatment reduced the body weight gain in OVX group around 35 and 23\%, respectively (Fig. 1). The PUAT/BW ratio was also decreased with both the treatments, UDCA $(-53 \%)$ and HRT $(-51 \%)$. The ratio liver/BW at sacrifice and food intake were not significantly different between the groups (Table 1).

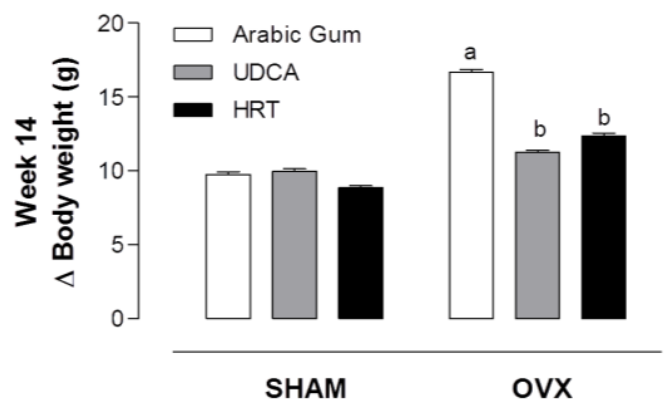

Figure 1 - Ovariectomy-induced bodyweight gain are attenuated by UDCA and HRT. Difference in bodyweight gain in SHAM and OVX mice and supplemented groups with either UDCA or HRT (during 4 weeks). Values are means \pm SEM of 8 experiments. ${ }^{a} \mathrm{p}<0.05$ significantly different from SHAM group and ${ }^{b}<0.05$ : significantly different from OVX group as identified by one-way ANOVA followed by a Tukey posttest.

Table 1 - Difference in food intake and the ratio of tissues to body weight.

\begin{tabular}{|c|c|c|c|c|c|c|}
\hline \multicolumn{7}{|c|}{ Food intake and tissue weight changes of female SHAM and OVX mice and treated groups with UDCA or HRT } \\
\hline Variable & SHAM & SHAM-UDCA & SHAM-HRT & OVX & OVX-UDCA & OVX-HRT \\
\hline $\begin{array}{c}\text { Food Intake } \\
(\text { g/dav/kg BW })\end{array}$ & $1006.6 \pm 55.51$ & $1071.2 \pm 55.87$ & $1036.7 \pm 30.62$ & $1138.4 \pm 68.53$ & $1008.1 \pm 76.17$ & $1019.4 \pm 76.98$ \\
\hline Uterus/BW (\%) & $0.11 \pm 0.01$ & $0.068 \pm 0.007$ & $0.083 \pm 0.009$ & $0.022 \pm 0.006^{\mathrm{a}}$ & $0.032 \pm 0.01^{\mathrm{a}}$ & $0.024 \pm 0.009^{\mathrm{a}}$ \\
\hline Liver/BW (\%) & $2.31 \pm 0.33$ & $2.8 \pm 0.52$ & $2.27 \pm 0.18$ & $3.05 \pm 0.28$ & $2.92 \pm 0.49$ & $2.62 \pm 0.58$ \\
\hline PUAT/BW (\%) & $1.60 \pm 0.14$ & $1.78 \pm 0.24$ & $1.60 \pm 0.34$ & $4.84 \pm 0.37^{\mathrm{a}}$ & $2.27 \pm 0.46^{\mathrm{b}}$ & $2.36 \pm 0.27^{\mathrm{b}}$ \\
\hline
\end{tabular}

Values are means \pm SEM of 8 experiments. ${ }^{a} \mathrm{p}<0.05$ significantly different from SHAM group and ${ }^{\mathrm{b}} \mathrm{p}<0.05$ : significantly different from OVX group as identified by one-way ANOVA followed by a Tukey posttest.

\section{Fasting Plasma and Serum Profiles}

Table 2 depicts the plasma and serum dosages of these animals. Although no differences was noted in the food intake between these animal groups, the fasting plasma glucose levels were significantly elevated in the OVX mice $(+37 \%)$, as well as total cholesterol $(+89 \%)$, LDL cholesterol levels $(+77 \%)$ and VLDL cholesterol levels $(+45 \%)$. UDCA-treatment reduced plasma glucose levels in $38 \%$ and reduced total cholesterol, LDL cholesterol and VLDL cholesterol levels in around 20, 17 and 19\%, respectively. Treatment with the hormone replacement therapy also reduced plasma glucose $(-23 \%)$, total cholesterol $(-17 \%)$, LDL cholesterol $(-37 \%)$ and VLDL cholesterol $(23 \%)$ levels. Average fasting, TG and HDL cholesterol levels were not significantly different between the groups. 
Table 2 - Biochemical analysis.

\begin{tabular}{ccccccc}
\hline \multicolumn{6}{c}{ Physiological measurements of female SHAM and OVX mice and treated groups with UDCA or HRT } \\
\hline Variable & SHAM & SHAM-UDCA & SHAM-HRT & OVX & OVX-UDCA & OVX-HRT \\
Glucose (mg/dL) & $120.8 \pm 1.44$ & $125.4 \pm 6.48$ & $118 \pm 6.16$ & $166 \pm 5.46^{\mathrm{a}}$ & $102 \pm 10.2^{\mathrm{b}}$ & $127 \pm 7.82^{\mathrm{b}}$ \\
Total cholesterol (mg/dL) & $148.7 \pm 14.61$ & $168.5 \pm 14.21$ & $203.2 \pm 11.52$ & $280.9 \pm 6.35^{\mathrm{a}}$ & $225.3 \pm 11.65^{\mathrm{b}}$ & $232.9 \pm 6.03^{\mathrm{b}}$ \\
HDL cholesterol (mg/dL) & $63.5 \pm 7.84$ & $58.9 \pm 1.33$ & $64.2 \pm 5.66$ & $68.1 \pm 2.93$ & $73.3 \pm 3.38$ & $72.5 \pm 7.07$ \\
LDL cholesterol (mg/dL) & $94.2 \pm 28$ & $114.6 \pm 38.2$ & $112.9 \pm 35.8$ & $166.6 \pm 22.9^{\mathrm{a}}$ & $108.9 \pm 22.13^{\mathrm{b}}$ & $103.9 \pm 15.8^{\mathrm{b}}$ \\
VLDL cholesterol (mg/dL) & $20.2 \pm 5.76$ & $21.3 \pm 3.19$ & $20.1 \pm 5.79$ & $29.4 \pm 5.88^{\mathrm{a}}$ & $23.6 \pm 1.4^{\mathrm{b}}$ & $22.6 \pm 2.06^{\mathrm{b}}$ \\
Triglycerides (mg/dL) & $48.5 \pm 7.5$ & $45.3 \pm 8.7$ & $44.6 \pm 3.3$ & $53.1 \pm 6.63$ & $49.3 \pm 0.7$ & $50.3 \pm 7.3$ \\
\hline
\end{tabular}

Values are means \pm SEM of 8 experiments. ${ }^{a} \mathrm{p}<0.05$ significantly different from SHAM group and ${ }^{\mathrm{b}} \mathrm{p}<0.05$ : significantly different from OVX group as identified by one-way ANOVA followed by a Tukey posttest.

\section{Liver and PUAT Histological Analysis and Liver Total Lipids Contents Measurements}

Histological analysis revealed that in contrast to the livers of SHAM mice (Fig. 2), the livers of OVX mice presented considerable amounts of lipid droplets. When quantified by the gravimetry, the livers of SHAM mice exhibited normal contents of total lipids of approximately 7.4\% (Adams and Angulo 2005), while livers from the OVX mice presented significantly higher values $(+154 \%)$ (Fig. 3). Both the treatments, UDCA and HRT, decreased lipid deposition in the liver of OVX mice around 28 and 55\% (Fig. 3), respectively, which was also observed in HE slices (Fig. 2). Visual analyses of the representative histological sections of PUAT (Fig. 4) showed that the heterogeneity in adipocyte size decreased from SHAM> OVX-UDCA > OVX-HRT > OVX: the highest number of smaller adipocytes was present in PUAT of SHAM mice.

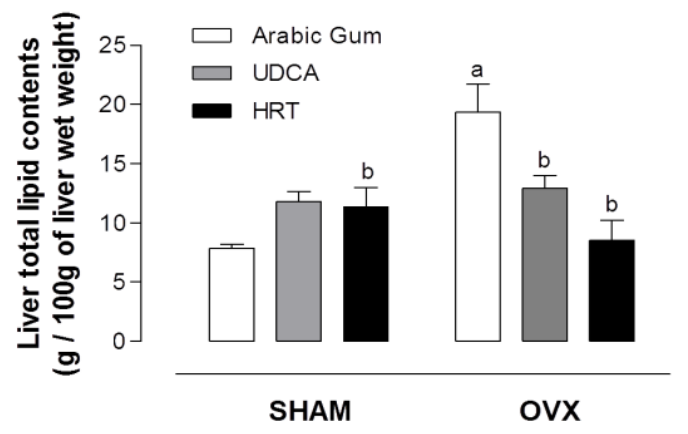

Figure 3 - Ovariectomy-variations of liver lipid deposition reduced by UDCA and HRT. Liver total lipid contents homogenates. Values are means \pm SEM of 8 experiments. ${ }^{\mathrm{a}} \mathrm{p}<0.05 \quad$ significantly different from SHAM group and ${ }_{\mathrm{p}}^{\mathrm{b}}<0.05$ : significantly different from OVX group as identified by one-way ANOVA followed by a Tukey posttest.
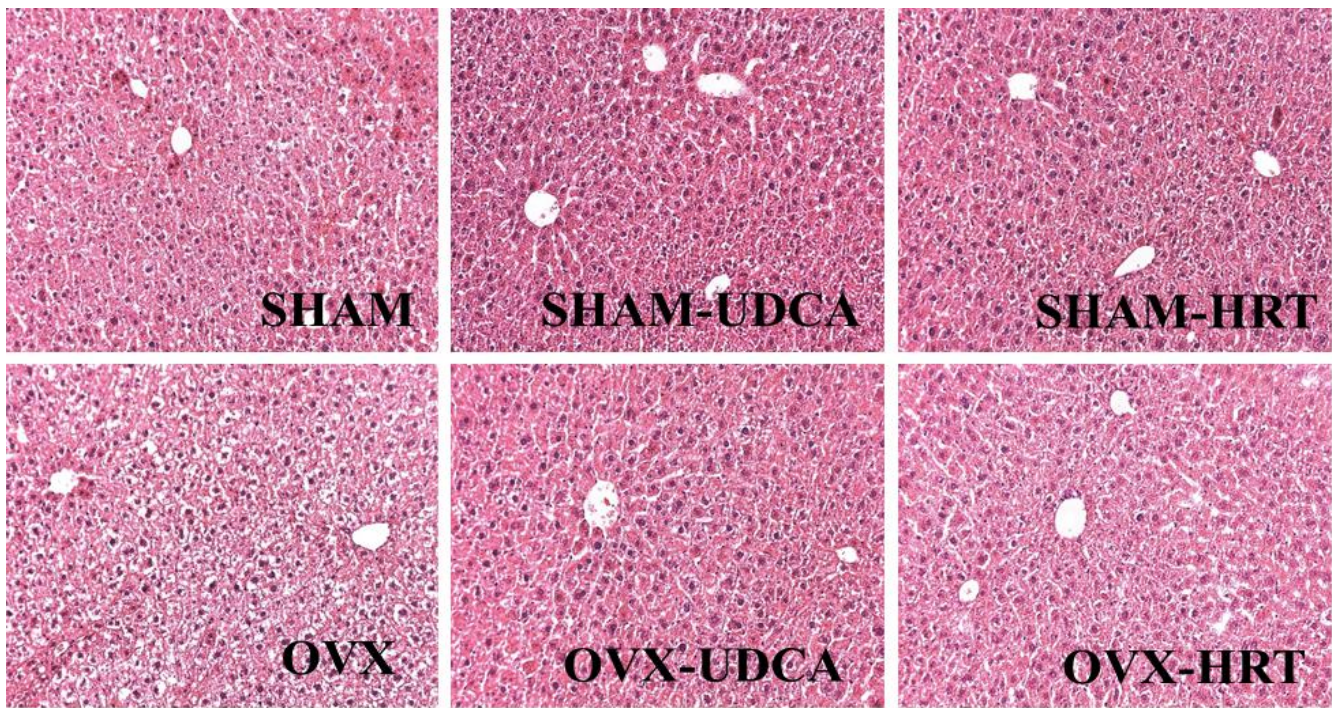

Figure 2 - Ovariectomy-variations and UDCA and HRT treatments in liver lipid deposition. Histological analyses of representative liver sections stained using hematoxylin and eosin (HE). Images captured at $40 \mathrm{x}$. 


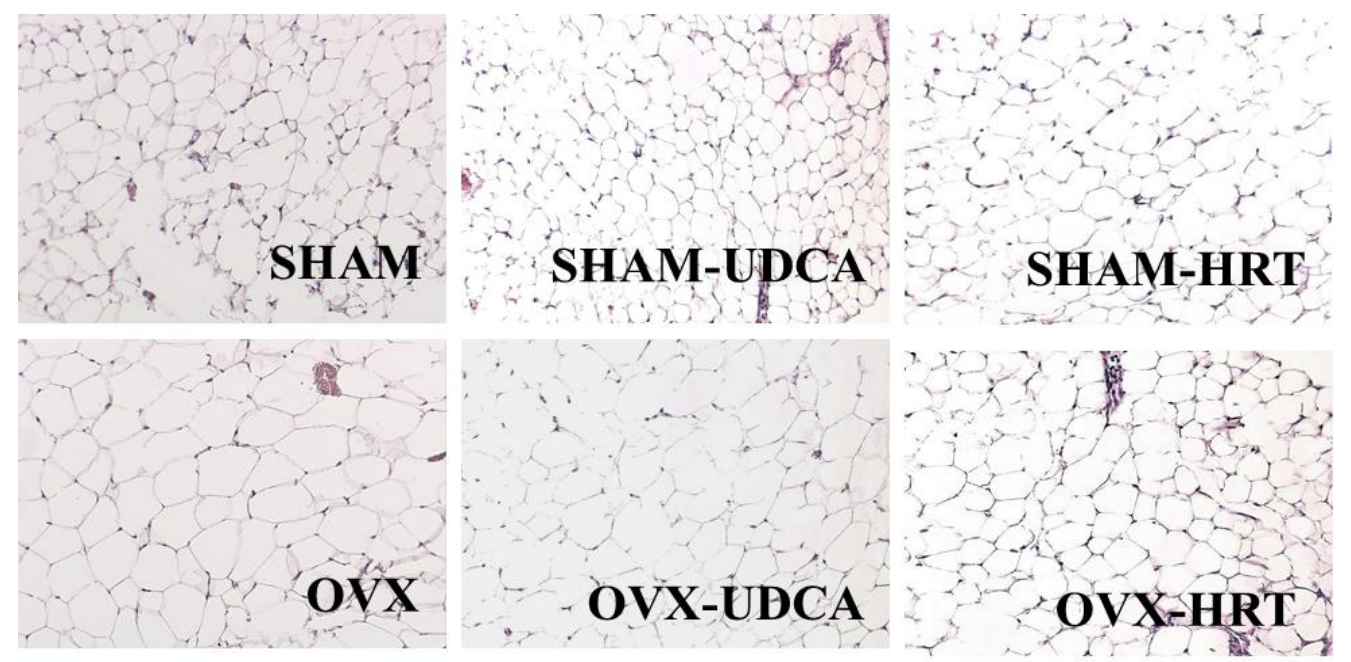

Figure 4 - Ovariectomy-variations and UDCA and HRT treatments in adipose tissue. Representative histological sections of PUAT stained using hematoxylin and eosin. Images captured at $40 \mathrm{x}$.

\section{DISCUSSION}

The present study assessed the use of UDCA as an alternative to estrogen replacement to correct postmenopausal metabolic changes in an ovariectomized mouse model. Results revealed that OVX mice, fed with a standard diet during 14 weeks after the surgery, reproduced a situation comparable to a physiological state of menopause that occurred at the end of fertile phase in women (Raafat et al. 2001). The levels of glucose, total cholesterol, low-density-lipoprotein (LDLcholesterol) and very-low-density-lipoprotein (VLDL-cholesterol) were higher in OVX mice than in SHAM mice and these animals also exhibited increased body weight and adiposity; similar results have been reported for rodents with estrogen deficiency and women (Bryzgalova et al. 2008; Rogers et al. 2009). As expected, the livers of these animals exhibited higher content of lipids in the liver than the mice from SHAM group as it was comproved by the histological analysis and by gravimetry measurements.

This lipid accumulation could be result of several factors, such as excessive food intake, alterations in uptake, or exportation of fatty acids, changes in the mitochondrial oxidation of fatty acids and a reduction in the export of triacylglycerols (Villanova et al. 2005). The average daily intake of food was not different between SHAM and OVX groups, indicating that lipid accumulation in OVX mice was not a result of hyperphagy. But probably a result of alterations in fatty acid metabolism, as an activated hepatic fatty acid synthesis and impaired fatty acid oxidation (Kumagai et al. 1993; Sanyal et al. 2001; Kim et al. 2010).

Body weight gain and adiposity were reduced with both the treatments and the levels of glucose, total cholesterol, LDL-cholesterol and VLDLcholesterol were decreased in OVX mice treated with UDCA, or HRT. The HRT is already known as a preventing treatment for weight gain and skin folds observed after menopause (Hassager and Christiansen 1989), as seen in the present results. HRT and UDCA treatment also had a positive result reducing the weight gain and adiposity. Besides, the reduced cholesterol results could be explained by its cytoprotective and immunomodulatory properties (Pageaux et al. 1995), which influenced the rate of cholesterol molecules and regulated the metabolism.

The livers of the treated animals exhibited lower lipid deposition than the mice from OVX group, as it was comproved by the histological analysis and by the gravimetric measurements. Adipose tissue inflammation and dysfunction are correlated with fatty acid 'overflow' to and subsequent triglyceride storage in peripheral organs such as muscle and liver (Tiniakos et al. 2010). OVX aggravated liver steatosis probably by an enhanced flow of fatty acids to the liver. Both, the UDCA and HRT treatment diminished OVX liver steatosis. Possible mechanisms by which this could be accomplished were direct effects (Lefebvre et al. 2009), as increased fatty acid oxidation in the liver and improved adipose tissue 
function that might result in less fatty acid overflow, thus lowering the hepatic steatosis.

\section{CONCLUSIONS}

In conclusion, UDCA treatment was shown to correct the disadvantageous metabolic changes associated with the loss of ovarian hormone production, as well as hormone replacement therapy. Both the treatments improved the body weight, liver steatosis and plasmatic parameters, showing a possible alternative treatment for the post-menopausal metabolic dysfunctions with ursodeoxycholic acid, a possibility that deserved further experimental investigation. These results would be useful for conduction "clinical trials" that tested the effects of UDCA in weight reduction, improvement of biochemical markers and liver disease on the patients.

\section{AUTHOR CONTRIBUTIONS}

The authors Angelo Christiano Franzoni, Aryane Martins Amorim and João Victor Mariano da Silva contributed equally to this work.

\section{REFERENCES}

Adams LA, Angulo P. Recent concepts in non-alcoholic fatty liver. Diabetes Med. 2005; 22: 1129-1133.

Anderson GL, Limacher M, Assaf AR, Tamsen Bassford MD, Beresford SAA, Black H, et al. Effects of conjugated equine estrogen in postmenopausal women with hysterectomy: the Women's Health Initiative randomized controlled trial. JAMA. 2004; 291: 1701-1712.

Beral V. Breast cancer and hormone-replacement therapy in the Million Women Study. The Lancet. 2003; 362: 419-427.

Bryzgalova G, Lundholm L, Portwood N, Gustafsson J, Khan A, Efendic S, et al. Mechanisms of antidiabetogenic and body weight-lowering effects of estrogen in high-fat diet-fed mice. Am J PhysiolEndoc M. 2008; 295(4): 904-912.

Colombo C, Battezzati PM, Podda M, Bettinardi $\mathrm{N}$, Giunta A. Ursodeoxycholic acid for liver disease associated with cystic fibrosis: a double-blind multicenter trial. The Italian Group for the Study of Ursodeoxycholic Acid in Cystic Fibrosis. Hepatology. 1996; 23(6): 1484-1490.

Cooke PS, Naaz A. Role of estrogens in adipocyte development and function. Exp Biol Med. 2004; 229(11): 1127-1135.
Folch J, Lees M, Sloane Stanley GH. A simple method for the isolation and purification of total lipids from animal tissues. J Biol Chem. 1957; 226: 497-509.

Hassager C, Christiansen C. Estrogen/gestagen therapy changes soft tissue body composition in postmenopausal women. Metabolism. 1989; 38(7): 662-665.

Hofmann AF. Pharmacology of ursodeoxycholic acid, an enterohepatic drug. Scand J Gastroenterol. 1994; 204: 1-15.

Hulley S, Grady D, Bush T, Furberg C, Herrington D, Riggs $\mathrm{B}$, et al. Randomized trial of estrogen plus progestin for secondary prevention of coronary heart disease in postmenopausal women. JAMA. 1998; 280: 605-613.

Keiding S, Höckerstedt $\mathrm{K}$, Bjøro $\mathrm{K}$, Bondesen S, Hjortrup A, Isoniemi $\mathrm{H}$, et al. The nordic multicentre double-blind randomized controlled trial of prophylactic ursodeoxycholic acid in liver transplant patients. Transplantation. 1997; 63: 1591-1594.

Kim YY, Kim SH, Oh S, Sul OJ, Lee HY, Kim HJ, et al. Increased fat due to estrogen deficiency induces bone loss by elevating monocyte chemoattractant protein (MCP-1) production. Molecular Cells. 2010; 29: 277-282.

Kumagai S, Holmäng A, Björntorp P. The effects of oestrogen and progesterone on insulin sensitivity in female rats. Acta Physiol Scand. 1993; 149: 91-97.

Lefebvre P, Cariou B, Lien F, Kuipers F, Staels B. Role of bile acids and bile acid receptors in metabolic regulation. Physiol Rev. 2009; 89(1): 147-191.

Lekander I, Borgstrom F, Strom O, Zethraeus N, Kanis JA. Cost-effectiveness of hormone replacement therapy for menopausal symptoms in the UK. Menopause Int. 2009; 15(1): 19-25.

Lobo RA. Metabolic syndrome after menopause and the role of hormones. Maturitas. 2008; 60(1): 10-18.

Pageaux GP, Blanc P, Perrigault PF, Navarro F, Fabre JM, Souche B, et al. Failure of ursodeoxycholic acid to prevent acute cellular rejection after liver transplantation. J Hepatol. 1995; 23: 119-122.

Raafat AM, Li S, Bennett JM, Hofseth LJ, Haslam SZ. Estrogen and estrogen plus progestin act directly on the mammary gland to increase proliferation in a post-menopausal mouse model. J Cell Physiol. 2001; 187(1): 81-89.

Reis CM, Melo NR, Meirelles ES, Vezozzo DP, Halpern A. Body composition, visceral fat distribution and fat oxidation in postmenopausal women using oral or transdermal oestrogen. Maturitas. 2003; 46(1): 59-68.

Rogers NH, Perfield II JW, Strissel KJ, Obin SM, Greenberg AS. Reduced energy expenditure and increased inflammation are early evens in the development of ovariectomy-induced obesity. Endocrinology. 2009; 150(5): 2161-2168. 
Rossouw JE, Anderson GL, Prentice RL, LaCroix AZ, Kooperberg C, Hutchinson F, et al. Risks and benefits of estrogen plus progestin in healthy postmenopausal women: principal results from the Women's Health Initiative randomized controlled trial. JAMA. 2002; 288: 321-333.

Sanyal AJ, Campbell-Sargent C, Mirshani F, Rizzo WB, Contos MJ, Sterling RK, et al. Nonalcoholic steatohepatitis: association of insulin resistance and mitochondrial abnormalities. Gastroenterology. 2001; 120: 1183-1192.

Schneider JG, Tompkins C, Blumenthal RS, Mora S. The metabolic syndrome in women. Cardiol Rev. 2006; 14(6): 286-291.
Suzuki A, Abdelmalek MF. Nonalcoholic fatty liver disease in women. Womens Health (Lond Engl). 2009; 5(2): 191-203.

Tiniakos DG, Vos MB, Brunt EM. Nonalcoholic fatty liver disease: pathology and pathogenesis. Annu Rev Pathol. 2010; 5: 145-171.

Villanova N, Zoli M, Marchesini G. Endothelial dysfunction and cardiovascular risk profile in nonalcoholic fatty liver disease. Hepatology. 2005; 42: 473-480.

Volpe A, Facchinetti F, Grasso A, Petraglia F, Campanini D, Genazzani AR. Benefits and risks of different hormonal replacement therapies in postmenopausal women. Maturitas. 1986; 8(4): 327-334. 